\title{
Neoadjuvant immunotherapy in non-small cell lung cancer: the sooner the better?
}

\author{
Jose Luis Perez-Gracia ${ }^{1,2,3}$, Miguel F. Sanmamed ${ }^{1,2}$, Ignacio Melero ${ }^{2,3,4}$ \\ ${ }^{1}$ Oncology Department, Clinica Universidad of Navarra (CUN), Pamplona, Spain; ${ }^{2}$ Health Research Institute of Navarra (IDISNA), Pamplona, \\ Spain; ${ }^{3}$ Centro de Investigación Biomédica en Red de Cáncer (CIBERONC), ${ }^{4}$ Department of Immunology and Cancer Immunotherapy, CUN and \\ CIMA, Pamplona, Spain \\ Correspondence to: Dr. Jose Luis Perez-Gracia. Oncology Department, Clinica Universidad of Navarra (CUN), Avda. Pio XII, 36,31008 Pamplona, \\ Spain. Email: jlgracia@unav.es. \\ Comment on: Cottrell TR, Thompson ED, Forde PM, et al. Pathologic features of response to neoadjuvant anti-PD-1 in resected non-small-cell lung \\ carcinoma: a proposal for quantitative immune-related pathologic response criteria (irPRC). Ann Oncol 2018;29:1853-60.
}

Submitted Oct 11, 2018. Accepted for publication Oct 22, 2018.

doi: $10.21037 /$ tlcr.2018.10.12

View this article at: http://dx.doi.org/10.21037/tlcr.2018.10.12

Recently, Forde et al. reported in the New England Fournal of Medicine a pilot study in which two neoadjuvant doses of the PD-1 inhibitor nivolumab were administered in a preoperative fashion to 21 patients with untreated, surgically resectable early (stage I-IIIA) non-small cell lung cancer (NSCLC) (1). Surgery was performed 4 weeks after the first dose of nivolumab. In addition to an acceptable toxicity profile, which did not cause delays in surgery, nivolumab induced a major pathological response in 9 of 20 resected tumors (45\%). Pathological responses were observed in patients presenting $\mathrm{PD}-\mathrm{L} 1$ positive and negative tumors and, interestingly, they correlated with pretreatment tumor mutational burden. The authors found that the number of T-cell clones observed in the tumor and peripheral blood increased following nivolumab in eight of nine patients. Remarkably, the authors described one patient presenting a complete response in which neoantigen-specific T-cell clones from the primary tumor rapidly expanded in peripheral blood following treatment. Moreover, some of these clones were not detected before treatment with nivolumab, thus suggesting that PD-1 blockade induced their expansion.

In a follow-up paper of the same study, Cottrell et al. evaluated the relationship between pathologic response to immunotherapy and long-term outcome (2). Such correlations have been evaluated for NSCLC patients treated with neoadjuvant chemotherapy $(3,4)$, but not with PD1 or PD-L1 blockade. For this purpose, they analyzed pre-treatment and post-treatment resection specimens of the 20 patients who underwent complete resection in this study, as well as the corresponding CT scans from the patients. They described that the regression bed, defined as the area of immune-mediated tumor clearance, was characterized by (I) immune activation-dense tumor infiltrating lymphocytes with macrophages and tertiary lymphoid structures; (II) massive tumor cell death-cholesterol clefts; and (III) tissue repair, neovascularization and proliferative fibrosis. All these features were more frequently observed in patients presenting major pathologic response, as compared with non-responders $(\mathrm{P}<0.05)$. The regression bed also explained the discrepancies that were described in the study between CT imaging and pathologic assessment of residual tumor. Finally, based on these findings, the authors developed 'Immune-Related Pathologic Response Criteria' (irPRC), which showed high reproducibility among pathologists.

As most cancer therapies, blockade of the PD1/PD-L1 pathway was initially explored in patients with advanced tumors that had been previously treated with standard therapies. Nevertheless, its success has moved this strategy forward to the first line scenario, and ultimately to the adjuvant setting. The benefit of adjuvant nivolumab in melanoma patients (5) which was superior to the CTLA-4 inhibitor ipilimumab, which had previously proved superiority over placebo in the adjuvant setting (6) and of durvalumab in patients with stage III NSCLC following radical chemoradiation (7) are representative examples of the benefits of this strategy.

The study by Forde and colleagues, published in the New England Fournal of Medicine, and the follow-up published in Annals of Oncology hold great value in both the patient care 
and translational research settings. Several ongoing studies are evaluating the activity of neoadjuvant immunotherapy in different tumor types, including NSCLC, urothelial cancer, melanoma, renal cancer, ovarian cancer, glioblastoma, gastrointestinal cancers or breast cancer, among others (www.clinicaltrials.gov). Arguments to justify a potentially higher activity of neoadjuvant over adjuvant immunotherapy include that the presence of the tumor may become a source of infiltrating immune cells and neoantigens that is absent in adjuvant strategies, since the tumor has been removed in the latter case (8). On the opposite hand, removal of the tumor may also eliminate a source of potential immunosuppressive factors, which may hamper the immune response. As for the translational research setting, neoadjuvant studies like this one allow to monitor molecular changes in sequential tumor samples, and therefore they represent a relevant source of knowledge on tumor biology (9).

The promising results of neoadjuvant immunotherapy reported by this study are in line with the preclinical data presented by Liu et al. (10). In this study, the authors confirmed the superiority of neoadjuvant over adjuvant immunotherapeutic strategies to eradicate lung metastasis in two model of spontaneous metastatic breast cancer in mice. It remains to be seen if the pathological responses observed in surgically amenable cases of NSCLC can be confirmed in NSCLC, and extended to other malignant diseases. Since a large number of adjuvant trials involving PD-1 and PD-L1 blockade are listed in Clinicaltrial.gov, the near future will be fertile in reports assessing these attractive opportunities to make the most of immunotherapy.

Finally, the development of robust and reproducible criteria to evaluate tumor response to neoadjuvant immunotherapy, like the Immune-Related Pathologic Response Criteria reported in this paper will allow a reliable evaluation of the activity of this strategy; to perform an accurate assessment of the prognosis of these patients; and to perform comparisons between different clinical studies performed in the neoadjuvant setting.

\section{Acknowledgements}

None.

\section{Footnote}

Conflicts of Interest: JL Perez-Gracia: consultant, research support, travel grants and lecture fees from BMS and Roche. Research support and travel grants from MSD. I Melero: consultant for BMS, Merck-Serono, Roche-Genentech, Tusk, Alligator, Genmab, Molecular Partners, F-Star, Bayer and research funding from BMS, Roche and Alligator. Dr. Sanmamed has no conflicts of interest to declare.

\section{References}

1. Forde PM, Chaft JE, Smith KN, et al. Neoadjuvant PD-1 Blockade in Resectable Lung Cancer. N Engl J Med 2018;378:1976-86.

2. Cottrell TR, Thompson ED, Forde PM, et al. Pathologic features of response to neoadjuvant anti-PD-1 in resected non-small-cell lung carcinoma: a proposal for quantitative immune-related pathologic response criteria (irPRC). Ann Oncol 2018;29:1853-60.

3. Yamane Y, Ishii G, Goto K, et al. A novel histopathological evaluation method predicting the outcome of non-small cell lung cancer treated by neoadjuvant therapy: the prognostic importance of the area of residual tumor. J Thorac Oncol 2010;5:49-55.

4. Liu-Jarin X, Stoopler MB, Raftopoulos H, et al. Histologic assessment of non-small cell lung carcinoma after neoadjuvant therapy. Mod Pathol 2003;16:1102-8.

5. Weber J, Mandala M, Del Vecchio M, et al. Adjuvant Nivolumab versus Ipilimumab in Resected Stage III or IV Melanoma. N Engl J Med 2017;377:1824-35.

6. Eggermont AM, Chiarion-Sileni V, Grob JJ, et al. Adjuvant ipilimumab versus placebo after complete resection of highrisk stage III melanoma (EORTC 18071): a randomised, double-blind, phase 3 trial. Lancet Oncol 2015;16:522-30.

7. Antonia SJ, Villegas A, Daniel D, et al. Durvalumab after Chemoradiotherapy in Stage III Non-Small-Cell Lung Cancer. N Engl J Med 2017;377:1919-29.

8. Melero I, Berraondo P, Rodríguez-Ruiz ME, et al. Making the Most of Cancer Surgery with Neoadjuvant Immunotherapy. Cancer Discov 2016;6:1312-4.

9. Perez-Gracia JL, Sanmamed MF, Bosch A, et al. Strategies to design clinical studies to identify predictive biomarkers in cancer research. Cancer Treat Rev 2017;53:79-97.

10. Liu J, Blake SJ, Yong MC, et al. Improved Efficacy of Neoadjuvant Compared to Adjuvant Immunotherapy to Eradicate Metastatic Disease. Cancer Discov 2016;6:1382-99.

Cite this article as: Perez-Gracia JL, Sanmamed MF, Melero I. Neoadjuvant immunotherapy in non-small cell lung cancer: the sooner the better? Transl Lung Cancer Res 2018;7(Suppl 4):S356S357. doi: 10.21037/tlcr.2018.10.12 